\title{
CEA-targeting Agent RG6123
}

National Cancer Institute

\section{Source}

National Cancer Institute. CEA-targeting Agent RG6123. NCI Thesaurus. Code C159892.

An agent targeting the tumor-associated antigen (TAA) carcinoembryonic antigen (CEA), with potential antineoplastic activity. Upon administration, CEA-targeting agent RG6123 targets and binds to human CEA that is specifically expressed on certain tumor cells. This may, through an as of yet not elucidated mechanism of action, kill CEA-expressing tumor cells. CEA is overexpressed in many cancer cell types. 